\title{
A Review of Physical Layer of Mobile WiMAX System and OFDM
}

\author{
Jayshree Sisodiya ${ }^{1}$, Deepti Rai ${ }^{2}$ \\ ${ }^{1}$ PG Student , ECE Deptt. , Alpine Institute of Technology, Ujjain \\ ${ }^{2}$ HOD , ECE Deptt. , Alpine Institute of Technology, Ujjain
}

\begin{abstract}
The WiMAX technology, based on the IEEE 802.16-2004 Air Interface Standard israpidly proving itself as a technology that will play a key role in fixed broadband wirelessmetropolitan area networks. The first certification lab, established at Cetecom Labs inMalaga, Spain is fully operational and more than 150 WiMAX trials are underway inEurope, Asia, Africa and North and South America. Unquestionably, Fixed WiMAX,based on the IEEE 802.16-2004 [1] Air Interface Standard, has proven to be a cost-effective fixed wireless alternative to cable and DSL services. In December, 2005 theIEEE ratified the 802.16e amendment [2] to the 802.16 standard. This amendment addsthe features and attributes to the standard necessary to support mobility. The WiMAXForum is now defining system performance and certification profiles based on the IEEE802.16e Mobile Amendment and, going beyond the air interface, the WiMAX Forum isdefining the network architecture necessary for implementing an end-to-end MobileWiMAX2 network. Release-1 system profiles will be completed in early 2006.This particular paper focusses on giving an overview of the mobile Wimax technology and LDPC coding typically used to enhance the performance of OFDM systems.
\end{abstract}

Keywords: OFDM, WiMAX ,Physical Layer, QoS , Circular prefix.

\section{Introduction to Mobile WiMAX}

Mobile WiMAX is a broadband wireless solution that enables convergence of mobile andfixed broadband networks through a common wide area broadband radio accesstechnology and flexible network architecture. The Mobile WiMAX Air Interface adoptsOrthogonal Frequency Division Multiple Access (OFDMA) for improved multipathperformance in non-line-of-sight environments. Scalable OFDMA (SOFDMA) [3] isintroduced in the IEEE 802.16e Amendment to support scalable channel bandwidths from1.25 to $20 \mathrm{MHz}$. The Mobile Technical Group (MTG) in the WiMAX Forum isdeveloping the Mobile WiMAX system profiles that will define the mandatory andoptional features of the IEEE standard that are necessary to build a Mobile WiMAX-compliant air interface that can be certified by the WiMAX Forum. The Mobile WiMAXSystem Profile enables mobile systems to be configured based on a common base featureset thus ensuring baseline functionality for terminals and base stations that are fullyinteroperable. Some elements of the base station profiles are specified as optional toprovide additional flexibility for deployment based on specific deployment scenarios thatmay require different configurations that are either capacity-optimized or coverage-optimized. Release-1 Mobile WiMAX profiles will cover 5, 7, 8.75, and $10 \mathrm{MHz}$ channelbandwidths for licensed worldwide spectrum allocations in the $2.3 \mathrm{GHz}, 2.5$ $\mathrm{GHz}, 3.3 \mathrm{GHz}$ and $3.5 \mathrm{GHz}$ frequency bands. The WiMAX Forum Network Working Group (NWG) is developing the higher-levelnetworking specifications [4] for Mobile WiMAX systems beyond what is defined in theIEEE 802.16 standard that simply addresses the air interface specifications. Thecombined effort of IEEE 802.16 and the WiMAX Forum help define the end-to-endsystem solution for a Mobile WiMAX network.Mobile WiMAX systems offer scalability in both radio access technology and networkarchitecture, thus providing a great deal of flexibility in network deployment options andservice offerings.
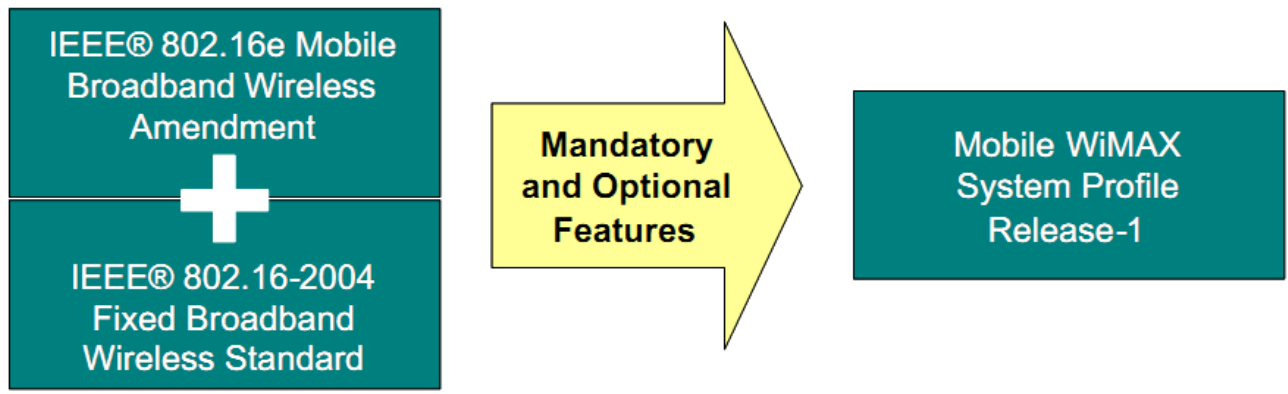

Figure 1: Mobile WiMAX system Profile

\section{Salient Features Supported by Mobile WiMAX}

Some of the salient features supported by Mobile WiMAX are:

- High Data Rates: The inclusion of MIMO antenna techniques along with flexible sub-channelization schemes, Advanced Coding and Modulation all enable the Mobile WiMAX technology to support peak DL data rates up to $63 \mathrm{Mbps}$ per sector and peak UL data rates up to $28 \mathrm{Mbps}$ per sector in a $10 \mathrm{MHz}$ channel.

- Quality of Service (QoS): The fundamental premise of the IEEE 802.16 MAC architecture is QoS. It defines Service Flows which can map to Diff Serv code points or MPLS flow labels that enable end-to-end IP based QoS.

Volume 6 Issue 7, July 2017 


\section{International Journal of Science and Research (IJSR) \\ ISSN (Online): 2319-7064}

Index Copernicus Value (2015): 78.96 | Impact Factor (2015): 6.391

Additionally, sub-channelization and MAP-based signaling schemes provide a flexible mechanism for optimal scheduling of space, frequency and time resources over the air interface on a frame-by-frame basis.

- Scalability: Despite an increasingly globalized economy, spectrum resources for wireless broadband worldwide are still quite disparate in its allocations. Mobile WiMAX technology therefore, is designed to be able to scale to work in different channelizations from 1.25 to $20 \mathrm{MHz}$ to comply with varied worldwide requirements as efforts proceed to achieve spectrum harmonization in the longer term. This also allows diverse economies to realize the multi-faceted benefits of the Mobile WiMAX technology for their specific geographic needs such as providing affordable internet access in rural settings versus enhancing the capacity of mobile broadband access in metro and suburban areas.

- Security: The features provided for Mobile WiMAX security aspects are best in class with EAP-based authentication, AES-CCM-based authenticated encryption, and CMAC and HMAC based control message protection schemes. Support for a diverse set of user credentials exists including; SIM/USIM cards, Smart Cards, Digital Certificates, and Username/Password schemes based on the relevant EAP methods for the credential type.

- Mobility: Mobile WiMAX supports optimized handover schemes with latencies less than 50 milliseconds to ensure real-time applications such as VoIP perform without service degradation. Flexible key management schemes assure that security is maintained during handover. While the Mobile WiMAX standards activity has been progressing, equipment suppliers have been aggressively developing equipment that will be WiMAX/802.16e-2005 compliant. With commercial availability of Mobile WiMAX-compliant equipment anticipated in the very near future and the launch of
WiBro services this year in Korea, it begs the question as to how the Mobile WiMAX technology relates to and impacts concurrent advances in $3 \mathrm{G}$ cellular technology. To address this question it is necessary to gain an understanding of the underlying technology for Mobile WiMAX as well as theplanned $3 \mathrm{G}$ enhancements.

\section{OFDMA basics for Mobile WiMAX}

Orthogonal Frequency Division Multiplexing (OFDM) [6,7] is a multiplexing techniquethat subdivides the bandwidth into multiple frequency sub-carriers as shown in Figure 2.In an OFDM system, the input data stream is divided into several parallel sub-streams ofreduced data rate (thus increased symbol duration) and each sub-stream is modulated andtransmitted on a separate orthogonal subcarrier. The increased symbol duration improvesthe robustness of OFDM to delay spread. Furthermore, the introduction of the cyclicprefix (CP) can completely eliminate Inter-Symbol Interference (ISI) as long as the CPduration is longer than the channel delay spread. The CP is typically a repetition of thelast samples of data portion of the block that is appended to the beginning of the datapayload as shown in Figure 3. The CP prevents interblock interference and makes thechannel appear circular and permits low-complexity frequency domain equalization.

A perceived drawback of $\mathrm{CP}$ is that it introduces overhead, which effectively reducesbandwidth efficiency. While the $\mathrm{CP}$ does reduce bandwidth efficiency somewhat, theimpact of the CP is similar to the "roll-off factor" in raised-cosine filtered single-carriersystems. Since OFDM has a very sharp, almost "brick-wall" spectrum, a large fraction ofthe allocated channel bandwidth can be utilized for data transmission, which helps tomoderate the loss in efficiency due to the cyclic prefix.

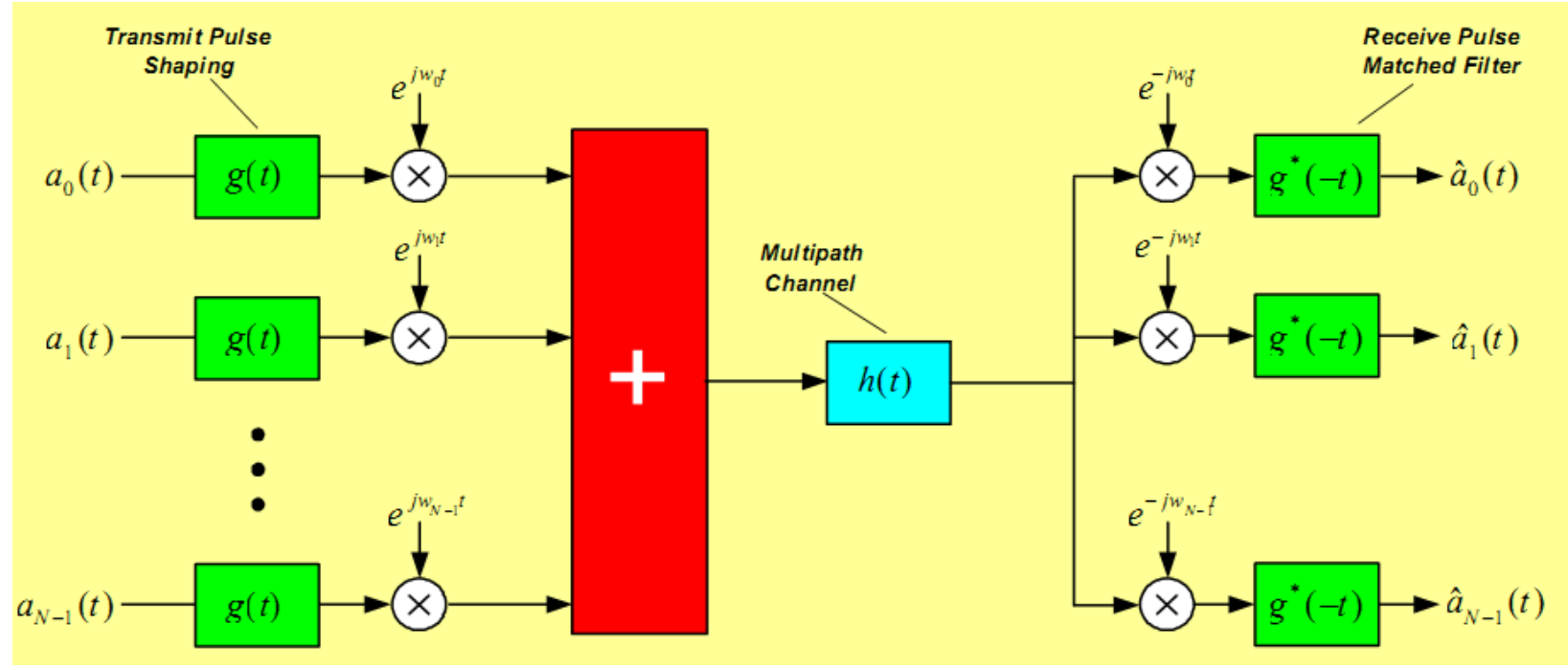

Figure 2: Basic Architecture of an OFDM System

OFDM exploits the frequency diversity of the multipath channel by coding andinterleaving the information across the sub-carriers prior to transmissions. OFDMmodulation can be realized with efficient Inverse Fast Fourier Transform (IFFT), whichenables a large number of sub-carriers (up to
2048) with low complexity. In an OFDMsystem, resources are available in the time domain by means of OFDM symbols and inthe frequency domain by means of subcarriers. The time and frequency resources can beorganized into sub-channels for allocation to individual users. 


\section{International Journal of Science and Research (IJSR) \\ ISSN (Online): 2319-7064 \\ Index Copernicus Value (2015): 78.96 | Impact Factor (2015): 6.391}

Orthogonal FrequencyDivision Multiple Access (OFDMA) is a multiple-access/multiplexing scheme thatprovides multiplexing operation of data streams from multiple users onto the downlinksub-channels and uplink multiple access by means of uplink sub-channels.

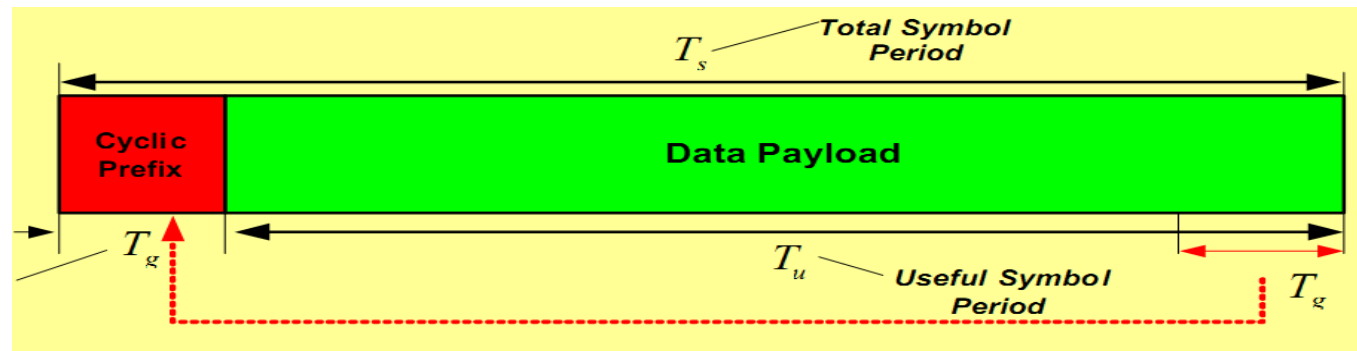

Figure 3: Insertion of Cyclic Prefix

\section{OFDMA Symbol Structure and Sub Chanellization}

The OFDMA symbol structure consists of three types of sub-carriers as shown in Figure4:

- Data sub-carriers for data transmission
- Pilot sub-carriers for estimation and synchronization purposes

- Null sub-carriers for no transmission; used for guard bands and DC carriers

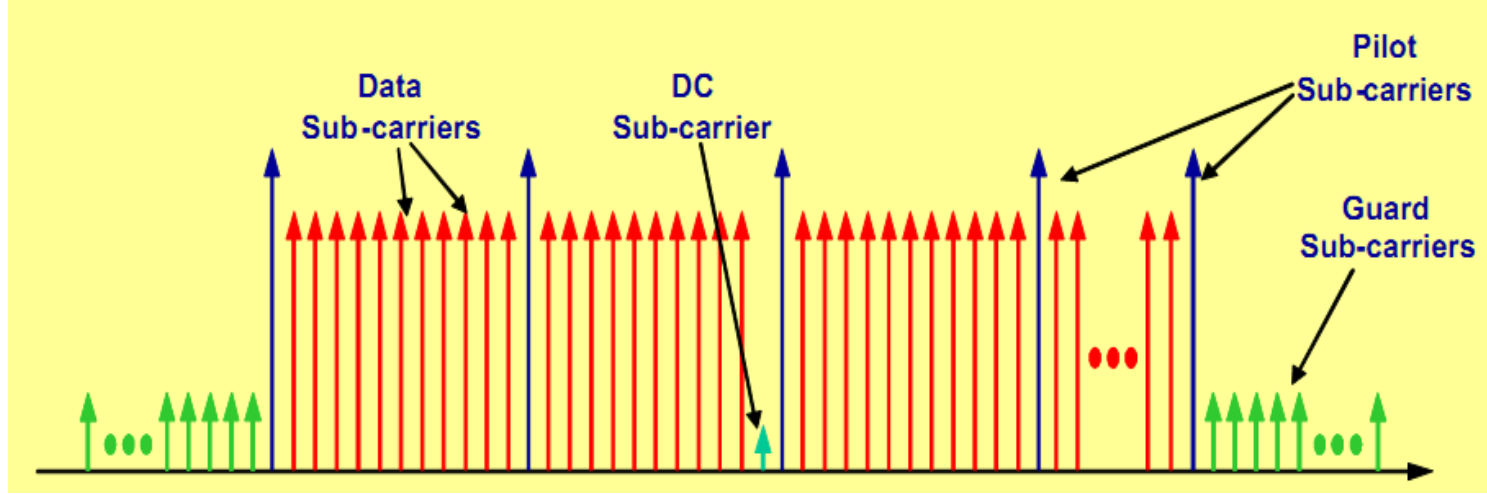

Figure 4: OFDMA Sub-Carrier Structure

Active (data and pilot) sub-carriers are grouped into subsets of sub-carriers called sub-channels. The WiMAX OFDMA PHY [3] supports sub-channelization in both DL andUL. The minimum frequency-time resource unit of subchannelization is one slot, whichis equal to 48 data tones (sub-carriers).There are two types of sub-carrier permutations for sub-channelization; diversity andcontiguous. The diversity permutation draws sub-carriers pseudo-randomly to form asub-channel. It provides frequency diversity and inter-cell interference averaging. Thediversity permutations include DL FUSC (Fully Used Sub-Carrier), DL PUSC (PartiallyUsed Sub-Carrier) and UL PUSC and additional optional permutations. With DL PUSC,for each pair of OFDM symbols, the available or usable sub-carriers are grouped intoclusters containing 14 contiguous sub-carriers per symbol period, with pilot and dataallocations in each cluster in the even and odd symbols as shown in Figure 5.

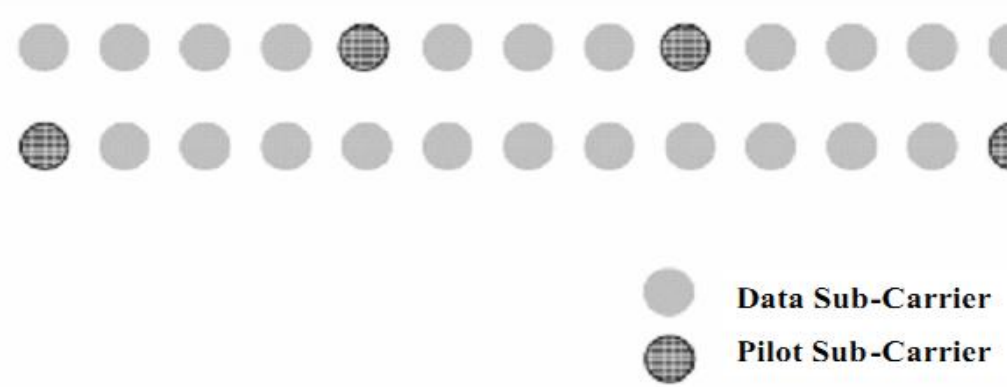

Figure 5: DL Frequency Diverse Sub-Channel

A re-arranging scheme is used to form groups of clusters such that each group is made upof clusters that are distributed throughout the sub-carrier space. A sub-channel in a groupcontains two (2) clusters and is made up of 48 data sub-carriers and eight (8) pilot sub-carriers. The data subcarriers in each group are further permutated to generate sub-channels within the group. Therefore, only the pilot positions in the cluster are shown inFigure 5. The data sub- 


\section{International Journal of Science and Research (IJSR) \\ ISSN (Online): 2319-7064}

Index Copernicus Value (2015): 78.96 | Impact Factor (2015): 6.391

carriers in the cluster are distributed to multiple subchannels.Analogous to the cluster structure for DL, a tile structure is defined for the UL PUSCwhose format is shown in Figure 6

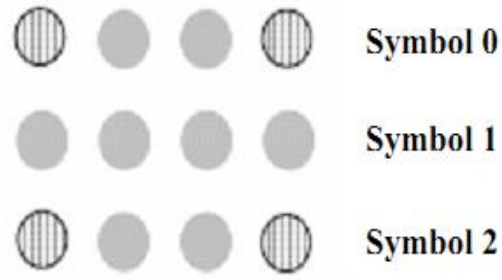

Pilot Sub-Carrier

\section{Data Sub-Carrier}

Figure 6: Tile Structure for UL PUSC

The available sub-carrier space is split into tiles and six (6) tiles, chosen from across theentire spectrum by means of a re-arranging/permutation scheme, are grouped together toform a slot. The slot comprises 48 data sub-carriers and 24 pilot sub-carriers in 3 OFDMsymbols. The contiguous permutation groups a block of contiguous sub-carriers to form a sub-channel. The contiguous permutations include DL AMC and UL AMC, and have thesame structure. A bin consists of 9 contiguous sub-carriers in a symbol, with 8 assignedfor data and one assigned for a pilot. A slot in AMC is defined as a collection of bins ofthe type ( $\mathrm{N} \times \mathrm{M}=6)$, where $\mathrm{N}$ is the number of contiguous bins and $\mathrm{M}$ is the number ofcontiguous symbols. Thus the allowed combinations are [( 6 bins, 1 symbol), ( 3 bins, 2 symbols), (2 bins, 3 symbols), (1 bin, 6 symbols)]. AMC permutation enables multi-userdiversity by choosing the sub-channel with the best frequency response.In general, diversity subcarrier permutations perform well in mobile applications whilecontiguous sub-carrier permutations are well suited for fixed, portable, or low mobilityenvironments. These options enable the system designer to trade-off mobility forthroughput.

\section{Other Advanced Phy Layer Features}

Adaptive modulation and coding (AMC), Hybrid Automatic Repeat Request (HARQ)and Fast Channel Feedback $(\mathrm{CQICH})$ were introduced with Mobile WiMAX to enhancecoverage and capacity for WiMAX in mobile applications.Support for QPSK, 16QAM and 64QAM are mandatory in the DL with Mobile WiMAX.In the UL, 64QAM is optional. Both Convolutional Code (CC) and Convolutional TurboCode (CTC) with variable code rate and repetition coding are supported. Block TurboCode and Low Density Parity Check Code (LDPC) are supported as optional features. Table 1 summarizes the coding and modulation schemes supported in the MobileWiMAX profile the optional UL codes and modulation are shown in italics.

Table 1: Supported Code and Modulations

\begin{tabular}{|l|l|c|c|}
\hline \multicolumn{2}{|c|}{} & DL & UL \\
\hline \multicolumn{2}{|l|}{ Modulation } & QPSK, 16QAM, 64QAM & QPSK,16QAM, 64QAM \\
\hline \multirow{2}{*}{\begin{tabular}{l} 
Code \\
\multirow{2}{*}{ Rate }
\end{tabular}} & CC & $1 / 2,2 / 3,3 / 4,5 / 6$ & $1 / 2,2 / 3,5 / 6$ \\
\cline { 2 - 4 } & CTC & $1 / 2,2 / 3,3 / 4,5 / 6$ & $1 / 2,2 / 3,5 / 6$ \\
\cline { 2 - 4 } & Repetition & $\mathrm{x} 2, \mathrm{x} 4, \mathrm{x} 6$ & $\mathrm{x} 2, \mathrm{x} 4, \mathrm{x} 6$ \\
\hline
\end{tabular}

The frame duration is 5 milliseconds. Each frame has 48 OFDMsymbols, with 44 OFDM symbols available for data transmission. The highlighted valuesindicate data rates for optional 64QAM in the UL.The base station scheduler determines the appropriate data rate (or burst profile) for eachburst allocation based on the buffer size, channel propagation conditions at the receiver,etc.

A Channel Quality Indicator (CQI) channel is utilized to provide channel-stateinformation from the user terminals to the base station scheduler. Relevant channelstateinformation can be fed back by the CQICH including: Physical CINR, effective CINR,MIMO mode selection and frequency selective sub-channel selection. With TDDimplementations, link adaptation can also take advantage of channel reciprocity toprovide a more accurate measure of the channel condition (such as sounding).Hybrid Auto Repeat Request (HARQ) is supported by Mobile WiMAX. HARQ isenabled using $\mathrm{N}$ channel "Stop and Wait" protocol which provides fast response to packeterrors and improves cell edge coverage. Chase Combining and optionally, IncrementalRedundancy are supported to further improve the reliability of the retransmission. Adedicated ACK channel is also provided in the uplink for HARQ ACK/NACK signaling.

Multi-channel HARQ operation is supported. Multi-channel stop-and-wait ARQ with asmall number of channels is an efficient, simple protocol that minimizes the memoryrequired for HARQ and stalling [8]. WiMAX provides signaling to allow fullyasynchronous operation. The asynchronous operation allows variable delay betweenretransmissions which gives more flexibility to the scheduler at the cost of additionaloverhead for each retransmission allocation. HARQ combined together with $\mathrm{CQICH}$ andAMC provides robust link adaptation in mobileenvironments at vehicular speeds inexcess of 120 $\mathrm{km} / \mathrm{hr}$.

\section{References}

[1] "Air Interface for Fixed Broadband Wireless Access Systems,” IEEE STD 802.16 - 2004, October, 2004.

\section{Volume 6 Issue 7, July 2017 www.ijsr.net}




\section{International Journal of Science and Research (IJSR) \\ ISSN (Online): 2319-7064}

Index Copernicus Value (2015): 78.96 | Impact Factor (2015): 6.391

[2] "Air Interface for Fixed and Mobile Broadband Wireless Access Systems," IEEE P802.16e/D12,February, 2005.

[3] Hassan Yagoobi, "Scalable OFDMA Physical Layer in IEEE 802.16 WirelessMAN", Intel TechnologyJournal, Vol 08, August 2004.

[4] "WiMAX End-to-End Network Systems Architecture Stage 2: Architecture Tenets, Reference odeland Reference Points," WiMAX Forum, December, 2005.

[5] "Mobile WiMAX - Part II: A Comparative Analysis", WiMAX Forum, 2006

[6] L.J. Cimini, "Analysis and Simulation of a Digital Mobile Channel Using Orthogonal FrequencyDivision Multiplexing," IEEE Trans. Comm., vol. COM-33, no. 7, pp 665-675, June 1985.

[7] Richard Van Nee and Ramjee Prasad, "OFDM for Wireless Multimedia Communications," Artech House, 2000.

[8] W. Xiao and R. Ratasuk, "Analysis of Hybrid ARQ with Link Adaptation", Proceedings of the AnnualAllerton Conference on Communications, Control, and Computing, pp. 1618-1619, Oct 2002.

[9] G. Nair, J. Chou, T. Madejski, K. Perycz, P. Putzolu and J. Sydir "IEEE 802.16 Medium Access Controland Service Provisioning", Intel Technology Journal, vol 08, August 2004.

[10]F. Wang, A. Ghosh, R. Love, K. Stewart et.al., "IEEE 802.16e System Performance-Analysis andSimulation Results", Proc. of PIMRC, Berlin, Germany, Sept. 2005.

[11] Philippe Duplessis, "HSOPA: Exploiting OFDM and MIMO to take UMTS beyond HSDPA/HSUPA" ,Nortel Technical Journal, Issue 2, July 2005.

[12] John Hoadley and Al Javed, "Overview: Technology Innovation for Wireless Broadband Access", NortelTechnical Journal, Issue 2, July 2005.

[13]John Liva and Titus Kwok-Yeung Lo, "Digital Beamforming in Wireless Communications," ArtechHouse Publishers, 1996.

[14] S.M. Alamouti, "A Simple Transmit Diversity Technique for Wireless Communications," IEEE Journalon Selected Areas in Communications, vol. 16, pp 1451-1458, October 1998.

[15] V. Tarokh, H. Jafarkhani and A. R. Calderbank, "Spacetime Block Codes from Orthogonal Designs,"IEEE Transactions on Information Theory, vol. 45, pp. 14561467, July 1999. 